\title{
Co-existence of benign gynecological tumors with endometriosis in a group of 1,000 women
}

\author{
CHAROULA MATALLIOTAKI ${ }^{1}$, MICHAIL MATALLIOTAKIS ${ }^{1}$, PANAGIOTIS IEROMONACHOU ${ }^{2}$, \\ GEORGE N. GOULIELMOS ${ }^{3}$, MARIA I. ZERVOU $^{3}$, AGGELOS LALIOTIS ${ }^{4}$, \\ DEMETRIOS A. SPANDIDOS ${ }^{5}$, AYDIN ARICI $^{6}$ and IOANNIS MATALLIOTAKIS ${ }^{1}$
}

\author{
Departments of ${ }^{1}$ Obstetrics and Gynecology, and ${ }^{2}$ Histopathology, Venizeleio General Hospital, 71409 Heraklion; \\ ${ }^{3}$ Section of Molecular Pathology and Human Genetics, Department of Internal Medicine, School of Medicine, \\ University of Crete, 71003 Heraklion, Crete, Greece; ${ }^{4}$ Department of General Surgery, Croydon University Hospital, \\ CR7 YE London, UK; ${ }^{5}$ Laboratory of Clinical Virology, Medical School, University of Crete, \\ 71003 Heraklion, Crete, Greece; ${ }^{6}$ Department of Obstetrics, Gynecology and Reproductive \\ Sciences, Yale University School of Medicine, New Haven, CT 06520, USA
}

Received August 28, 2017; Accepted October 30, 2017

DOI: $10.3892 / \mathrm{ol} .2017 .7449$

\begin{abstract}
The purpose of this study was two-fold, first to investigate the association between endometriosis and the risk of benign gynecologic tumors and, secondly, to evaluate the distribution of endometrioma and ovarian cysts in women with endometriosis. The medical and pathological reports of 1,000 women with endometriosis were retrospectively reviewed. The incidence of ovarian cysts, uterine leiomyomas and adenomyosis, as well as the side of ovarian cysts were further compared. A total of 295 cases of endometriomas, 172 cases of adenomyosis, 173 cases of ovarian cysts and 89 cases of uterine leiomyomas were confirmed histologically in patients with endometriosis. Serous cysts represented the most frequent diagnosis $(n=81,8.1 \%)$ in women with ovarian cysts, followed by dermoid cysts $(n=15,1.2 \%)$. In women with unilateral endometriomas, the observed proportion of left-sided cysts was found in $65.6 \%$ (164 of 250), significantly higher compared with right-sided cysts (86 out of 250, 34.4\%) $(\mathrm{P}<0.001)$. Moreover, patients with other ovarian cysts were recognized as left-sided in $60 \%$ (96 out of 160) of cases, significantly higher compared with right-sided cysts (64 out of $160,40 \%)(\mathrm{P}<0.01)$. On the whole, the current study indicates that endometriosis may be associated with an increased risk of benign gynecological tumors, such as ovarian cysts, adenomyosis and leiomyomas. The results of this study confirm a left lateral predisposition of endometriomas and ovarian cysts.
\end{abstract}

Correspondence to: Dr Michail Matalliotakis, Department of Obstetrics and Gynecology, Venizeleio and Pananio General Hospital of Heraklion, Knossos Avenue, 71409 Heraklion, Greece

E-mail: mihalismat@hotmail.com

Key words: endometriosis, benign gynecological tumors, left lateral predisposition

\section{Introduction}

Endometriosis is a common, yet enigmatic gynecological disorder affecting 3-43\% women of reproductive age. Endometrioma is defined as the formation of an ovarian cyst with epithelial lining resembling the ectopic endometrium. It is observed in 17-44\% of women suffering from endometriosis and represents $35 \%$ of benign ovarian cysts requiring surgery $(1,2)$. Ovarian endometriomas are the third most common site for the manifestation of endometriosis after the pouch of Douglas and uterosacral ligament (2). They are associated with an advanced stage of endometriosis involving simultaneously other pelvic or abdominal structures, as exclusive ovarian disease is found in only $1.06 \%$ of 1,785 patients with endometriosis (3). Ovarian cysts and uterine leiomyomas represent a major source of gynecological morbidity in women of reproductive age. Both conditions are included among the top 5 leading causes of hospitalization for gynecological conditions unrelated to pregnancy in women aged between 15 and 44 years (4). Leiomyoma is characterized by uterine fibroids that are non-cancerous growths of muscle tissue in the uterus (and occasionally nearby) (5). Nowadays, it is believed that dysregulated mechanistic target of rapamycin (mTOR) signaling is a major component of leiomyoma etiology, considering that an activated mTOR signaling pathway is essential for fibroid growth (6).

Adenomyosis is a common gynecological disorder which is not yet well understood; it is a myometrial lesion characterized by the presence of ectopic endometrial glands and stroma located deep within the surrounding myometrium with adjacent myometrial hyperplasia and hypertrophy (7). For over 90 years, endometriosis and adenomyosis were considered as the same entity with the exception of endometriomas. The prevalence of adenomyosis ranges from 10 to $70 \%$ according to the applied diagnostic criteria (8). It is more often observed in women aged between 30-50 years. According to Vercellini et al (8), adenomyosis can be identified in 30-60\% of cases after hysterectomy, 
with the majority of females being multiparous. Adenomyosis is observed in 69-79\% of cases diagnosed with endometriosis, but only in $35-55 \%$ of women diagnosed with myomas. It has been found that adenomyosis is associated with an increased risk of endometrial hyperplasia, endometrial polyps or endometrial cancer (8).

We have previously reported a left lateral predisposition of endometrioma in two different countries (9). The limited number of studies and the small number of case reports published thus far, prompted us to investigate the association between benign gynecological tumors and endometriosis, and to further evaluate the distribution of left- and right-sided ovarian cysts in women with endometriosis.

\section{Patients and methods}

The records of all patients with endometriosis treated at the Yale University Hospital from 1995 to 2005 and from the Departments of Obstetrics and Gynecology of the University Hospital of Crete and Venizeleio General Hospital from 1990 to 2016, were reviewed. In total, 1,000 women with endometriosis who underwent surgical treatment by laparoscopy or laparotomy in two different geographical locations were investigated. The data were collected by clinicians and pathologists. The stage of endometriosis was scored according to the revised classification of the American Fertility Society (10). The clinicopathological characteristics of the benign gynecological tumors were further classified as previously described (11).

The study protocol was approved by the human subjects review boards at the two field centers. The Human Committee of Yale University School of Medicine approved the study for this evaluation (HIC \#12590), as well as the Department of Obstetrics and Gynecology of the University of Crete and the Obstetrics and Gynecology of the Venizeleio General Hospital of Crete. The Ethics Committee for Human Research of Venizeleio Hospital also approved the study (ECHR \#46/6686). All patients provided written informed consent prior to participation.

Data were recorded, including age, symptoms, stage of endometriosis, side (location, left or right side) and classification of cysts. Moreover, the information of the histological type of benign gynecological tumors was obtained from pathological records. In cases where two or more cysts were presented in the same gonad, we included the cases with the largest diameter (8). According to the findings, the patients were divided into 4 groups as follows: i) Ovarian or paraovarian cysts; ii) uterine leiomyomas; iii) adenomyosis; and iv) combination of all the above. The results are reported as the means \pm standard deviation or as percentages where appropriate. The frequency of left- and right-sided ovarian cancer was analyzed using the $\chi^{2}$ test to compare observed and external events. A $\mathrm{P}$-value $<0.05$ was considered to indicate a statistically significant difference.

\section{Results}

Among the 1,000 females with endometriosis, endometriomas were mostly observed $(29.5 \%)$, followed by adenomyosis and uterine leiomyomas (17.2 and 8.9\%), respectively (Table I).
Table I. Confirmation of benign gynecological conditions in 1,000 women with endometriosis ${ }^{\mathrm{a}}$.

\begin{tabular}{lc}
\hline Condition & No. $(\%)$ \\
\hline Ovarian or paraovarian of cyst & \\
Endometrioma & $295(29.5)$ \\
Serous cysts & $81(8.1)$ \\
Mucinous cysts & $14(1.4)$ \\
Dermoid cysts & $15(1.5)$ \\
Brenner tumors & $13(1.3)$ \\
Miscellaneous & $28(2.8)$ \\
Paraovarian cysts & $22(2.2)$ \\
Endometrioma and other cysts & $11(1.1)$ \\
Uterine leiomyomas & $89(8.9)$ \\
Adenomyosis & $172(17.2)$ \\
Endometrial polyps & $16(1.6)$ \\
Adenomyosis and leiomyomas & $11(11.1)$ \\
Leiomyomas and cysts & $16(1.6)$ \\
Adenomyosis and cysts & $16(1.6)$ \\
Adenomyosis, leiomyomas and cysts & $15(1.5)$ \\
\hline
\end{tabular}

Out of the 1,000 women diagnosed with endometriosis, 914 were confirmed to suffer from benign conditions. The remaining women, had endometriosis with other (not benign) conditions.

The patients with adenomyosis were the oldest $(42 \pm 3.6$ years) (Table II). The clinical characteristics of all women are presented in Table II. Infertility (47.8\%) was mostly observed in women with endometriomas. Additionally, adnexal mass (69.4\%) was found in patients with ovarian cysts and furthermore, pelvic pain was found mostly (53.5\%) in women with adenomyosis. In the patients with unilateral endometriomas, the observed proportion of left-sided cysts was found in 65.6\% (164/250), significantly greater compared with right-sided cysts $34.4 \%(86 / 250)\left(\chi^{2}=31.2 ; \mathrm{P}<0.001\right)$ and significantly different from the expected incidence of $50 \%(\mathrm{P}<0.001)$ (Table II). Additionally, other ovarian cysts in women with endometriosis were detected left-sided in $60 \%(96 / 160)$ of cases, notably higher compared with right-sided cysts $(64 / 160,40 \%)(\mathrm{P}<0.01)$ and significantly different from the expected incidence of $50 \%(\mathrm{P}<0.01)$ (Table II). Of not, in the cases with ovarian cysts, the serous cyst was the most common (8.1\%), followed by dermoid (1.5\%) (Table I).

\section{Discussion}

Endometriosis, ovarian cysts, adenomyosis and uterine myomas are benign diseases that commonly affect women of reproductive age. Inflammatory, environmental and genetic factors play a role in the development of these benign tumors and sometimes may be found in the same women $(12,13)$.

Previous studies have suggested that a comorbidity association exists between endometriosis and many benign gynecological tumors $(4,9,12,13)$, although the results of certain studies differ and are unexpected. Recently, Mahnert et al (12) proposed that 
Table II. Clinical characteristics and lateral distribution of endometrioma and ovarian cysts in the women studied.

\begin{tabular}{|c|c|c|c|c|}
\hline Characteristic & $\begin{array}{l}\text { Endometrioma } \\
\mathrm{n}=295(29.5 \% \text { of total } \\
\text { no. of women studied })\end{array}$ & $\begin{array}{l}\text { Ovarian cysts } \\
\mathrm{n}=173(17.3 \% \text { of total } \\
\text { no. of women studied })\end{array}$ & $\begin{array}{c}\text { Leiomyomas } \\
\mathrm{n}=89(8.9 \%) \text { of total } \\
\text { no. of women studied })\end{array}$ & $\begin{array}{c}\text { Ademyosis } \\
\mathrm{n}=172(17.2 \% \text { of total } \\
\text { no. of women studied })\end{array}$ \\
\hline Age (years) & $36 \pm 5.8$ & $38 \pm 6.2$ & $41 \pm 4.5$ & $42 \pm 3.6$ \\
\hline \multicolumn{5}{|c|}{ Main complaints (\%) } \\
\hline Pelvic pain & $120(40.6 \%)$ & $40(19.7 \%)$ & $35(39.2 \%)$ & $92(53.5 \%)$ \\
\hline Infertility & $141(47.8 \%)$ & $12(5.9 \%)$ & $9(10.1 \%)$ & $62(36 \%)$ \\
\hline Adnexal mass & $34(11.6 \%)$ & $141(69.4 \%)$ & $45(50.7 \%)$ & $18(10.5 \%)$ \\
\hline Left-sided & $164 / 250(65.6 \%)$ & $96 / 160(60 \%)$ & & \\
\hline Right-sided & $86 / 250(34.4 \%)$ & $64 / 160(40 \%)$ & & \\
\hline Bilateral & 45 & 15 & & \\
\hline
\end{tabular}

Values are presented as the means \pm standard deviation or $\mathrm{n}(\%)$.

benign pre-operative surgical indications including pelvic mass, endometriosis, pelvic pain or leiomyomas were found in $2.7 \%$ of unexpected gynecological malignancies. Furthermore, Verit and Yucel (13) in a meta-analysis indicated that endometriosis, leiomyomas and adenomyosis may be associated with an increased risk of gynecological cancers, such as endometrioma and ovarian cancers. Therefore, it has been proposed that ovarian endometriomas, in most cases (90\%), are formed by the invagination of the ovarian cortex. Although there seems to be a consensus regarding the invagination theory, there is still a controversy between the implantation theory and the metaplasia theory. However, an article published by Nisolle and Donnez (14), suggested that the mesothelium covering the ovary may invaginate into the ovarian cortex. Motta et al (15) also described invaginations of the mesothelial layer covering the ovarian surface. These mesothelian inclusions may be transformed into intra-ovarian endometriosis by metaplasia, under the influence of unknown factors.

The complexity of the disease and the limited progress in identifying its exact cause explain the reason for the existence of so many controversies in the literature regarding the most effective modality to treat endometriomas. In our series, we observed that endometriomas represented the most common type $(29.5 \%)$, followed by ovarian cysts $(20.3 \%)$, adenomyosis $(17.2 \%)$ and uterine leiomyomas $(8.9 \%)$. The current data indicate that women with endometriosis should be counseled about the future risks of developing these benign gynecological diseases. To the best of our knowledge, this is the first study on 1,000 women with endometriosis and the simultaneous co-existence of benign gynecological tumors. Despite the various theories developed to explain the establishment of endometriosis, there is a great deal of evidence to suggest that endometriosis is result of a poly-parametric system with multiple origins. In the current study, we found unilateral endometrioma was more frequent on the left-sided (65.6\%) than the right-sided (34.4\%) ovary. Moreover, in women with other ovarian cysts, the location site was were observed to be mostly on the left side $(60 \%)$, significantly higher compared with the right side $(40 \%)$.

Our results are in agreement with those of our previous study, in which it was reported that the majority of endometriomas were located in the left ovary. In that study, we suggested a new mechanical theory of implication, the female varicocele theory, which could play an important role in the development of ovarian endometriosis or endometriomas (9). These and previous results are in accordance with those of other studies (16-18), where it is observed that the left side of the pelvis is more frequently affected, possibly as the presence of the sigmoid colon reduces peritoneal fluid movement.

Considering that endometriosis arises from the interplay between genetic and environmental factors, various studies (described below) have attempted to elucidate whether any shared genetic factors exist for endometriosis, leiomyoma and adenomyosis or the genetic basis is very specific for each condition.

Various scenarios have been suggested thus far regarding the histogenesis of adenomyosis and the molecular pathways involved in this procedure. Thus, a study focusing on adenomyosis tissue reported that specific uterine marker molecules are expressed, such as oxytocin receptor (OTR), vasopressin receptor (VPR), estrogen receptor (ER) and progesterone receptor (PR) (19). Considering that angiogenesis represents an important factor in the development of adenomyosis, various growth factors, as well as a genetic polymorphism of fibroblast growth factor $2(F G F-2)$ have been suggested to be associated with the development of this condition (20). Moreover, the loss of heterozygosity ( $\mathrm{LOH})$ in DNA mismatch repair genes in adenomyosis has been reported by our group previously (21). A difference has been observed regarding the expression levels of ER and PR between the adenomyosis and leiomyoma. Of note, it was found that ER- $\beta$ expression and the lack of PR expression lead to the development of adenomyosis (22). Metalloproteinases (MMPs) consist a group of enzymes that mediate the degradation of most extracellular matrix proteins during organogenesis, growth and normal tissue turnover. Whilst their very low expression and activity in normal adult tissues (23), in patients with leiomyoma and/or adenomyosis MMPs as well as some specific cytokines have been found at significantly elevated levels (24).

The peroxisome proliferator-activated receptor- $\gamma(P P A R-\gamma)$ $161 \mathrm{CC}$ genotype was found to be a risk factor for adenomyosis 
and endometriosis, but not for leiomyoma (25). The $3 \mathrm{bp} \mathrm{I/D}$ polymorphism of the CYP19 gene showed an association with endometriosis, but not with the adenomyosis/leiomyoma subgroup (26). The $+2073 \mathrm{~A} / \mathrm{T}$ polymorphism of $E G F R$ gene (27) and the ACE I/D polymorphism (28) were found to be associated significantly with endometriosis and leiomyoma. Furthermore, the VEGF -1154GG genotype (29), two MMP-1 polymorphisms (30) and the $-181 \mathrm{~A} / \mathrm{G}$ polymorphism of MMP-7 (31) were found to be associated with endometriosis and adenomyosis.

Taken together, the aforementioned findings point out that some genetic factors may exhibit a pleiotropic effect concerning these conditions, thus being common risk factors for more than one of them.

In conclusion, the present study demonstrates that endometriosis is linked with an increased risk of benign gynecological tumors, such as ovarian cysts, adenomyosis and uterine leiomyomas. Additionally, the present data confirmed a left lateral predisposition of endometrium and other ovarian cysts. Overall, further research and investigations are required to elucidate the correlation between endometriosis and benign gynecological morbidities.

\section{Acknowledgements}

The authors would like to thank all the clinicians and the pathologists for providing the data and pathology reports used in this study.

\section{References}

1. Bedaiwy MA, Alfaraj S, Yong P and Casper R: New developments in the medical treatment of endometriosis. Fertil Steril 107: 555-565, 2017.

2. Busacca M and Vignali M: Ovarian endometriosis: From pathogenesis to surgical treatment. Curr Opin Obstet Gynecol 15: 321-326, 2003.

3. Redwine DB: Ovarian endometriosis: A marker for more extensive pelvic and intestinal disease. Fertil Steril 72: 310-315, 1999.

4. Velebil P, Wingo PA, Xia Z, Wilcox LS and Peterson HB: Rate of hospitalization for gynecologic disorders among reproductive-age women in the United States. Obstet Gynecol 86: 764-769, 1995.

5. Shozu M, Murakami K and Inoue M: Aromatase and leiomyoma of the uterus. Semin Reprod Med 22: 51-60, 2004.

6. Al-Hendy A, Diamond MP, Boyer TG and Halder SK: Vitamin D3 inhibits Wnt/ $\beta$-catenin and mTOR signaling pathways in human uterine fibroid cells. J Clin Endocrinol Metab 101: 1542-1551, 2016.

7. Ferenczy A: Pathophysiology of adenomyosis. Hum Reprod Update 4: 312-322, 1998.

8. Vercellini P, Viganò P, Somigliana E, Daguati R, Abbiati A and Fedele L: Adenomyosis: Epidemiological factors. Best Pract Res Clin Obstet Gynaecol 20: 465-477, 2006.

9. Matalliotakis IM, Cakmak H, Koumantakis EE, Margariti A Neonaki M and Goumenou AG: Arguments for a left lateral predisposition of endometrioma. Fertil Steril 91: 975-978, 2009.

10. American Society for Reproductive: Revised American Society for Reproductive Medicine classification of endometriosis: 1996 Fertil Steril 67: 817-821, 1997.

11. Russell P: Surface epithelial-stromal tumors of the ovary. In: Blaustein's Pathology of the Female Genital Tract. Kurman RJ (ed). 4th edition. Springer, New York, NY, pp705-782, 1994.

12. Mahnert N, Morgan D, Campbell D, Johnston C and As-Sanie S: Unexpected gynecologic malignancy diagnosed after hysterectomy performed for benign indications. Obstet Gynecol 125: 397-405, 2015.

13. Verit FF and Yucel O: Endometriosis, leiomyoma and adenomyosis: The risk of gynecologic malignancy. Asian Pac J Cancer Prev 14: 5589-5597, 2013.
14. Nisolle M and Donnez J: Peritoneal endometriosis, ovarian endometriosis, and adenomyotic nodules of the rectovaginal septum are three different entities. Fertil Steril 68: 585-596, 1997.

15. Motta PM, Van Blerkom J and Mekabe S: Changes in the surface morphology of ovarian germinal epithelium during the reproductive life and in some pathological conditions. J Submicrosc Cytol 99: 664-667, 1992.

16. Jenkins S, Olive DL and Haney AF: Endometriosis: Pathogenetic implications of the anatomic distribution. Obstet Gynecol 67: 335-338, 1986.

17. Vercellini P, Aimi G, De Giorgi O, Maddalena S, Carinelli S and Crosignani PG: Is cystic ovarian endometriosis an asymmetric disease? Br J Obstet Gynaecol 105: 1018-1021, 1998.

18. Al-Fozan H and Tulandi T: Left lateral predisposition of endometriosis and endometrioma. Obstet Gynecol 101: 164-166, 2003.

19. Mechsner S, Grum B, Gericke C, Loddenkemper C, Dudenhausen JW and Ebert AD: Possible roles of oxytocin receptor and vasopressin-1 $\alpha$ receptor in the pathomechanism of dysperistalsis and dysmenorrhea in patients with adenomyosis uteri. Fertil Steril 94: 2541-2546, 2010.

20. Kang S, Li SZ, Wang N, Zhou RM, Wang T, Wang DJ, Li XF, Bui J and Li Y: Association between genetic polymorphisms in fibroblast growth factor (FGF)1 and FGF2 and risk of endometriosis and adenomyosis in Chinese women. Hum Reprod 25: 1806-1811, 2010.

21. Goumenou AG, Arvanitis DA, Matalliotakis IM, Koumantakis EE and Spandidos DA: Loss of heterozygosity in adenomyosis on $h M S H 2, h M L H 1, p 16^{1 n k 4}$ and GALT loci. Int J Mol Med 6: 667-671, 2000.

22. Mehasseb MK, Panchal R, Taylor AH, Brown L, Bell SC and Habiba M: Estrogen and progesterone receptor isoform distribution through the menstrual cycle in uteri with and without adenomyosis. Fertil Steril 95: 2228-2235, 2235.e1, 2011.

23. Sorsa T, Tjäderhane L and Salo T: Matrix metalloproteinases (MMPs) in oral diseases. Oral Dis 10: 311-318, 2004.

24. Inagaki N, Ung L, Otani T, Wilkinson D and Lopata A: Uterine cavity matrix metalloproteinases and cytokines in patients with leiomyoma, adenomyosis or endometrial polyp. Eur J Obstet Gynecol Reprod Biol 111: 197-203, 2003.

25. Kiyomizu M, Kitawaki J, Obayashi H, Ohta M, Koshiba H, Ishihara $\mathrm{H}$ and Honjo $\mathrm{H}$ : Association of two polymorphisms in the peroxisome proliferator-activated receptor-gamma gene with adenomyosis, endometriosis, and leiomyomata in Japanese women. J Soc Gynecol Investig 13: 372-377, 2006.

26. Kado N, Kitawaki J, Obayashi H, Ishihara H, Koshiba H, Kusuki I, Tsukamoto K, Hasegawa G, Nakamura N, Yoshikawa T and Honjo H: Association of the CYP17 gene and CYP19 gene polymorphisms with risk of endometriosis in Japanese women. Hum Reprod 17: 897-902, 2002.

27. Hsieh YY, Chang CC, Tsai FJ, Lin CC and Tsai CH: T homozygote and allele of epidermal growth factor receptor 2073 gene polymorphism are associated with higher susceptibility to endometriosis and leiomyomas. Fertil Steril 83: 796-799, 2005.

28. Hsieh YY, Lee CC, Chang CC, Wang YK, Yeh LS and Lin CS: Angiotensin I-converting enzyme insertion-related genotypes and allele are associated with higher susceptibility of endometriosis and leiomyoma. Mol Reprod Dev 74: 808-814, 2007.

29. Liu Q, Li Y, Zhao J, Zhou RM, Wang N, Sun DL, Duan YN and Kang S: Association of single nucleotide polymorphisms in VEGF gene with the risk of endometriosis and adenomyosis. Zhonghua Yi Xue Yi Chuan Xue Za Zhi 26: 165-169, 2009 (In Chinese).

30. Kang S, Wang Y, Zhang JH, Jin X, Fang SM and Li Y: Single nucleotide polymorphism in the matrix metalloproteinases promoter is associated with susceptibility to endometriosis and adenomyosis. Zhonghua Fu Chan Ke Za Zhi 40: 601-604, 2005 (In Chinese).

31. Shan K, Lian-Fu Z, Hui D, Wei G, Na W, Xia J and Yan L: Polymorphisms in the promoter regions of the matrix metalloproteinases-7, -9 and the risk of endometriosis and adenomyosis in China. Mol Hum Reprod 12: 35-39, 2006.

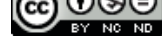

This work is licensed under a Creative Commons Attribution-NonCommercial-NoDerivatives 4.0 International (CC BY-NC-ND 4.0) License. 